\title{
A microarray analysis of genes involved in relating egg production to nutritional intake in Drosophila melanogaster
}

\author{
$\mathrm{J} \mathrm{Terashima}^{\star, 1}$ and M Bownes ${ }^{1}$ \\ 1 Institute of Cell Biology, School of Biology, University of Edinburgh, UK \\ * Corresponding author: J Terashima, Institute of Cell Biology, University of \\ Edinburgh, D713, Darwin Building, Kings Buildings, Mayfield Road, Edinburgh, \\ UK. Tel: + 44(0) 131650 5368; Fax: + 44(0) 131650 5371; \\ E-mail: jun.terashima@ed.ac.uk
}

Received 23.11.04; revised 12.1.05; accepted 13.1.05; published online 18.3.05 Edited by J Abrams

\begin{abstract}
Egg chambers of Drosophila are reabsorbed under conditions of nutritional shortage by inducing apoptosis at stages 8 and 9 , midway through oogenesis. Nutritional shortage leads to an increase in ecdysone concentration in flies. Apoptosis at stage $8 / 9$ is also induced by 20 -hydroxyecdysone injection into the females maintained with adequate nutrition. The expression pattern in the ovary of some ecdysone response genes, $E 75 A, B R-C$, is different according to the nutritional environment and the overexpression of these genes induces apoptosis. Apoptosis is suppressed by Juvenile hormone analog treatment of females under nutritional shortage. We predict nutritional and stress response genes control hormone levels and the increase in ecdysone concentration in the flies following starvation induces the ovarian apoptosis. We therefore used a microarray approach to identify the genes involved in receiving the nutritional signal from the environment and translating it in the ovary, thus initiating and executing apoptosis.

Cell Death and Differentiation (2005) 12, 429-440.

doi:10.1038/sj.cdd.4401587

Published online 18 March 2005
\end{abstract}

Keywords: apoptosis; microarray; drosophila; oogenesis; stress

Abbreviations: 20E, 20-hydroxyecdysone; JHA, juvenile hormone analogue

\section{Introduction}

Egg laying in Drosophila is closely linked to food availability. With adequate availability of nutrients and appropriate laying substrates, mated females of Drosophila melanogaster can lay up to half of their body weight a day as eggs. However, following nutrient withdrawal, the female responds rapidly and egg laying almost ceases. Increased cyst death in region $2 a / b$ of the germarium occurs very early oogenesis in response to nutritional shortage. ${ }^{1}$ In addition, mature eggs are frequently retained by the female, young previtellogenic egg chambers do not enter vitellogenesis and some egg chambers in early vitellogenesis undergo apoptosis. ${ }^{2,3}$ Those egg chambers that have completed vitellogenesis, complete chorion synthesis but are not laid. Subsequently, the female reduces production of yolk proteins in the fat body, but many of the early responses to the change in food conditions are in the ovary itself. ${ }^{3,4}$ Under adequate nutrition, the nurse cell nuclei in the egg chamber show DNA fragmentation from stage 12 of oogenesis, following the completion of cytoplasmic transfer from the nurse cells to the oocyte. ${ }^{5}$ Some reaper, hid and grim including genes crucial for the apoptosis in Drosophila have been identified, ${ }^{6-8}$ but they are not involved in the apoptosis of the nurse cells of egg chamber which commences at stage 12 during normal development. ${ }^{5}$

There seems to be a checkpoint or control point at stage 8/9 of oogenesis, ${ }^{9}$ just as vitellogenesis begins, when individual egg chambers make the decision to either develop or undergo apoptosis. The link between nutrients and the ovary seems to be mediated by the balance of the two key hormones, ecdysone and juvenile hormone $(\mathrm{JH}){ }^{2,10}$ We have recently shown that both hormones are crucial in the decision-making process in the egg chamber and they do this by modulating early ecdysone response genes including Broad-Complex $(B R-C) .{ }^{3} B R-C$ has four isoforms, $Z 1, Z 2, Z 3$ and $Z 4$, encoding a family of zinc-finger transcription factors ${ }^{11}$ and control oogenesis either by inducing apoptosis or controlling yolk protein gene expression and hence key developmental choices. ${ }^{3}$ The $B R-C$ isoform expression pattern is different in the ovary of females kept under nutritional shortage and those kept with adequate nutrition. $B R-C Z 2$ and $Z 3$ are not expressed in egg chambers at the stage 8 developmental checkpoint under adequate nutrition, but under nutritional shortage, both isoforms are expressed in the egg chamber at this stage. ${ }^{3}$ These expression patterns are possibly controlled by ecdysone concentration that is higher in the females under nutritional shortage than in the females under adequate nutrition. ${ }^{10}$ It seems likely that the expression of some genes, which can modulate hormone levels, are affected by nutritional status and consequently change and control the ecdysone concentration in the females. In addition, to actually induce apoptosis at stages 8 and 9, genes that control and select the apoptosis pathway should be induced. Some of these may be known stress response genes, since lack of nutrients will be a stress to the physiology of the female. Diverse stimuli, such as nutritional shortage and cytotoxic chemicals, can induce the apoptosis at mid-oogenesis (reviewed by McCall ${ }^{12}$ ). The nurse cells are the first cells to show signs of apoptosis. ${ }^{3,12}$

To investigate this process further and understand more of the genes involved in this switch between development and apoptosis, we undertook a microarray analysis. A model for the relationship between nutritional conditions and egg development is shown in Figure 1 (based on Terashima and 
Bownes $^{3}$ ). Our experiments aim to identify some of the genes in the ovary needed to execute the decision as to whether an individual egg chamber should undergo development into a mature egg or die by apoptosis. When the females are maintained under adequate nutrition, the flies produce many eggs. However, when the flies are maintained under starvation or when 20-hydroxyecdysone (20E) is injected into the abdomen of the females under adequate nutrition, an increase in apoptosis in egg chambers at stages 8 and 9 of oogenesis is observed (Figure 1, Table $1^{3}$ ). The apoptosis induced by starvation is suppressed by But juvenile hormone analog $(\mathrm{JHA})$ treatment of the abdomen of the females. ${ }^{2,3}$ For the microarray analysis, we used 3-day-old flies. This is because hormone levels fluctuate during the first days following eclosion as metamorphosis is completed. We therefore selected those flies that were mature and already producing viable eggs.

Our first comparison was therefore between the ovaries of starved and fed females. JH can partially rescue the apoptosis induced by starvation, ${ }^{2,3}$ so we compared the ovaries of starved females with and without $\mathrm{JH}$ analogue (JHA, methoprene) treatment. Finally, since $20 \mathrm{E}$ can mimic to some degree the effect of starvation by inducing apoptosis of egg

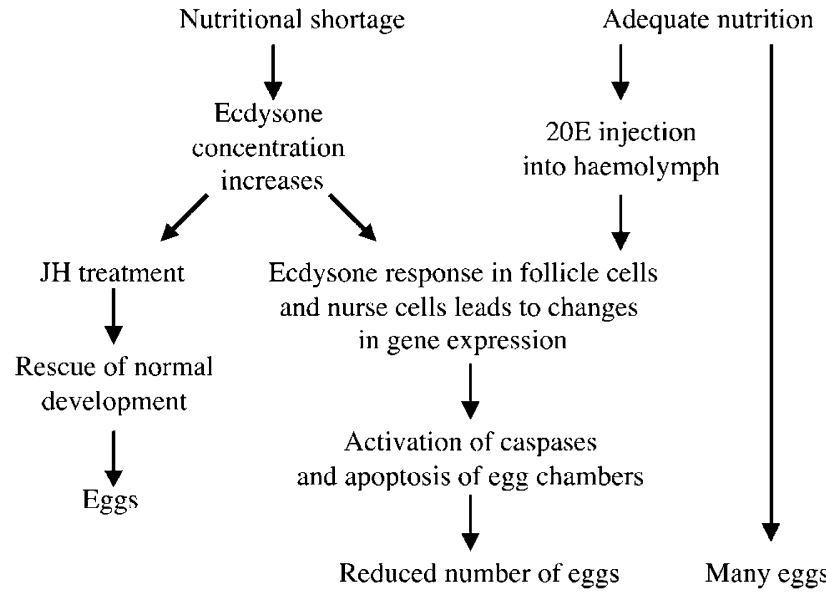

Figure 1 A model for the apoptosis pathway activated by nutritional shortage. The scheme proposes a model for the interaction of nutrition, ecdysone and apoptosis. Apoptosis is induced in the egg chambers at stages 8 and 9 under nutritional shortage and in $20 \mathrm{E}$-treated flies chambers, ${ }^{2,3}$ we next compared the ovaries of fed females with ovaries from fed females treated with $20 \mathrm{E}$.

In this paper, we describe some of the groups of genes that showed altered expression in the ovary and are likely to be involved in the execution of this survival/death developmental decision. We selected from the results genes in several categories including nutrient-sensing genes, stress response genes, genes capable of modulating hormone levels and apoptosis-related genes that may be involved in this process. In addition, we carried out in situ hybridization and RT-PCR for some genes to check their expression in the ovary and investigate if it matched the microarray data. In this way, we compared gene expression patterns under two sets of apoptotic conditions and two sets of conditions that promote development.

\section{Results}

Table 2 shows a summary of the results for the set of cDNAs used in the microarray and ovarian tissues. We carried out the microarray on a chip with 5364 genes (the list of genes on the chip is presented on http://www.ebi.ac.uk/arrayexpress. We carried out comparisons as follows: the ovary of starved flies and fed flies (S/F), JHA (methoprene)-treated starved flies and starved flies (JH/S) and 20E-treated fed flies and fed flies $(E / F)$. The aim was to determine which genes are up- or

Table 2 Comparison of the numbers of identified genes and their relative expression levels between various apoptotic conditions

\begin{tabular}{|l|c|c|c|}
\hline $\begin{array}{l}\text { Experimental } \\
\text { conditions compared }\end{array}$ & Upregulated & No change & Downregulated \\
\hline Starved/fed (S/F) & 2604 & 1385 & 1375 \\
\hline $\begin{array}{l}\text { JHA-treated/starved } \\
\text { (JH/S) }\end{array}$ & 1900 & 973 & 2491 \\
\hline $\begin{array}{l}\text { 20E-treated/fed } \\
\text { (E/F) }\end{array}$ & 2469 & 953 & 1942 \\
\hline
\end{tabular}

The table indicates the numbers of genes that upregulated, downregulated or remain unchanged in each combination of starved / fed (S/F), JHA-treated/ starved (JH/S) and 20E-injected/fed (E/F). Upregulated (red column) shows that gene expression increases under those conditions. Upregulation means that gene expression is activated by starvation $(S / F)$ and $20 E$ injection $(E / F)$ and $\mathrm{JHA}$ application (JH/S). Nochange (yellow column) indicates that gene expression is not changed with different experimental conditions. Downregulated (green column) indicates that gene expression is lower.

Table 1 Number of egg chamber at each stage and percentage of apoptosis under various condition

\begin{tabular}{|c|c|c|c|c|c|c|c|c|}
\hline & \multicolumn{6}{|c|}{ Number of egg chambers (S.D.) } & \multicolumn{2}{|c|}{$\%$ Apoptosis } \\
\hline & Stage 5 & Stage 6 & Stage 7 & Stage 8 & Stage 9 & Stage 10 & Stage 8 & Stage 9 \\
\hline Fed & 31.4 (3.4) & $20.8(4.3)$ & $27.6(2.8)$ & $16.4(2.9)$ & $21.1(1.8)$ & $19.9(1.8)$ & 1.5 & 5.5 \\
\hline Starved & *23.8 (2.7) & $19.3(2.9)$ & $22.1(3.5)$ & *11.3 (1.2) & *10.9 (2.8) & *3.4 (1.8) & *15.5 & 35.2 \\
\hline 20E-treated & $32.9(2.2)$ & $21.3(1.9)$ & $22.2(2.7)$ & *2.9 (2.1) & *3.9 (1.9) & ${ }^{\star} 0.5(0.9)$ & *82.9 & *90.3 \\
\hline Ringer-treated & $31.5(4.2)$ & $22.6(4.3)$ & $26.5(5.2)$ & $17.3(2.5)$ & $20.9(2.3)$ & $21.2(2.5)$ & 1.5 & 4.6 \\
\hline JHA-treated & *32.5 (4.1) & $20.5(4.2)$ & $22.0(3.1)$ & *16.3 (1.9) & *17.9 (2.1) & ${ }^{\star} 8.7(4.2)$ & ${ }^{\star} 8.2$ & 16.5 \\
\hline Acetone-treated & $24.6(4.9)$ & $21.3(2.8)$ & $24.3(4.6)$ & 10.1 (1.1) & 9.8 (1.5) & $3.6(2.5)$ & 15.2 & 35.6 \\
\hline
\end{tabular}

Significant difference (at $5 \%$ level, comparing fed and starved, 20E-treated and Ringers-treated, and JHA-treated and Acetone-treated). \% of apoptosis is calculated as follows: $\%=$ (mean of egg chamber in which apoptosis is induced/mean of total number of egg chambers) $\times 100(n=12)$. The table shows the number of egg chambers at each of the stages from 5 to 10 and the percentage of apoptosis at stages 8 and 9 in ovaries of fed, starved and $20 \mathrm{E}$ treated, Ringer-treated (control for 20E-treated), JHA-treated and acetone-treated (control for JHA-treated) flies. Nuclear condensation and fragmentation were detected by Hoechst staining and observed under a fluorescent microscope. *indicates significant differences within $5 \%$ ( $n=12$ flies). 
downregulated by starvation, $\mathrm{JH}$ and $20 \mathrm{E}$, respectively. We predicted that genes involved in apoptosis should be upregulated by starvation and $20 \mathrm{E}$ treatment and downregulated by JHA treatment, because $20 \mathrm{E}$ injection into fed flies and JHA treatment of starved flies induces and suppresses, respectively, the apoptosis of egg chambers at stages 8 and 9. ${ }^{2,3}$ Further, the $20 \mathrm{E}$ concentration in starved flies is higher than the concentration in fed flies ${ }^{10}$ (Terashima J, Takaki K, Sakurai S, and Bownes M, unpublished data).

The increase of $20 \mathrm{E}$ concentration during starvation and the induction of the apoptosis by $B R-C$ Z2, Z3 and E75A (Terashima and Bownes ${ }^{3}$ and unpublished data), which are ecdysone response genes, suggest that the higher levels of apoptosis observed during starvation are likely to be controlled indirectly by $20 \mathrm{E}$ concentration. Any genes in the ovary that control or modulate hormone titre should be up- or downregulated by starvation. We did not know whether any of these genes would be altered in their expression levels in the ovary itself as other tissues of the fly could equally well modulate hormone levels under different nutritional conditions. However, it is also possible that some genes in the ovary receive the starvation signals via other mechanisms and not through hormone regulation. Therefore, we analysed 2604 genes that are upregulated by starvation and 1375 genes that are downregulated by starvation. We expect these to include nutritional and stress response genes that regulate hormone synthesis or metabolism. In addition, we carried out RT-PCR and in situ hybridization to confirm expression levels and observe where and when the genes are expressed in the ovary. Some RT-PCR results did not reflect the results of the microarray analysis. This is likely to be because we used the whole ovary for the microarray. However, for the RT-PCR, we wanted to see what was happening prior to and at the checkpoint in oogenesis, thus we extracted total RNA from all the egg chambers up to stage 9 or from just stage 8 and 9 egg chambers. The different results between RT-PCR and microarray analysis is therefore likely to be caused by removing the egg chamber after stage 10 . This removes them in the next stage of analysis as we move from the microarray to the more detailed analysis of gene expression in genes which are simply upregulated as late development proceeds.

Rather than report on long lists of random genes, we are making the full data set available on http://www.ebi.ac.uk/ arrayexpress. We have grouped some of the affected genes, which are known to be involved in pathways whose expression we expect to be altered by nutrition and which alter hormone levels. We will concentrate in this manuscript on those genes which may enable us to better understand the role of pathways that are at least partially known and which we propose are involved in relating environmental cues to reproduction.

\section{Nutrition- and stress-related genes}

One of the aspects of the decision to undergo development or apoptosis is that cells need to be induced to undergo cell proliferation or proliferation needs to be inhibited, respectively. We predict that there are nutritional response and stress response pathways upstream of the pathways that modulate hormone levels. However, we have no idea how many of the enzymes that can lead to ecdysone concentration increases will operate in the ovary itself as opposed to other tissues of the fly. We did not know if the ovary itself was capable of modifying hormone levels either in the ovary itself or affect haemolymph levels. takeout (to) and basket (bsk) are starvation and stress response genes, respectively. ${ }^{13,14}$ bsk encodes c-jun $\mathrm{N}$ terminal kinase (JNK), ${ }^{14}$ which induces apoptosis in the Drosophila wing ${ }^{15}$ and neural tissues through activation of Plenty of $\mathrm{SH} 3 \mathrm{~s}(\mathrm{POSH}) .{ }^{16}$ Higher expression of to, bsk and $P O S H$ in the ovary were induced by starvation (Figure 2a). bskand POSHexpression were downregulated in the $\mathrm{JH} / \mathrm{S}$ combination. Higher POSH expression was induced by $20 \mathrm{E}$ application, but bsk was not affected by $20 \mathrm{E}$. to expression was upregulated by $\mathrm{JHA}$ treatment $(\mathrm{JH} / \mathrm{S})$ and downregulated by $20 \mathrm{E}$ treatment (E/F). RT-PCR also detected higher expression in starved (compared with fed), but differences in expression levels between starvation and JHA treatment was not detected by RT-PCR (Figure 2b). We propose that to expression was enhanced after stage 10 in oocyte development and suppressed in stage $10 \mathrm{egg}$ chambers under starvation. We thought that bsk and $\mathrm{POSH}$ could induce the apoptosis observed in stage 8 and 9 egg chambers. Therefore, we checked expression levels in the egg chambers at stages 8 and 9 (Figure 2b). bsk and $\mathrm{POSH}$ expression reflected the results of the microarray analysis; starvation induced higher expression of bsk and $\mathrm{POSH}$ in the ovary prior to stage 10 and in the egg chambers at stages 8 and 9. 20E induced higher expression of $\mathrm{POSH}$ but not bsk, and JHA treatment suppressed the expression of both genes in the ovary prior to stage 10 and in the egg chambers at stages 8 and 9 (Figure 2b). Starvation and stress must be sensed in the ovary and the first step in responding to starvation may well involve alterations in the expression of these genes. We expect that expression of these genes will be clearly upregulated by starvation, and are upregulated or remain unchanged following $20 \mathrm{E}$ treatment Therefore, to, bsk and $\mathrm{POSH}$ are candidates for sensing starvation and subsequently affecting the progress of oocyte development.

The mechanism of body size control in Drosophila is described by Potter and $\mathrm{Xu}{ }^{17}$ and Oldham and Hafen. ${ }^{18}$ The scheme in Figure 2c shows the relationship between some of the genes involved in cell growth in response to nutrition and their relationship to the insulin signalling pathway (based on Potter and $\mathrm{Xu},{ }^{17}$ and Oldham and Hafen ${ }^{18}$ ). Target of rapamysin (Tor) responds to nutrition and induces cell growth through activation of RPS60-p70-protein kinase (S6k). S6k mutants have a smaller body size than wild-type flies. ${ }^{17}$ Simultaneous activation of S6k and TOR suppresses the Drosophila homologue of $4 \mathrm{E}-\mathrm{BP}^{19}$ and Thor negatively regulates body size in Drosophila. ${ }^{20}$ TOR and S6k are inactivated by Tuberous Sclerosis Complex 1 (TSC1)/TSC2, which induces cell proliferation. ${ }^{17,18}$ To induce cell growth, TSC1/TSC2 is inhibited by Protein kinase $\mathrm{B}(A k t)$, which is activated by the insulin receptor through activation of Dstpk61 (the Drosophila homologue of PDK1). ${ }^{21}$ If insulin binds to the insulin receptor, the receptor activates AKT through Dstpk61 and AKT inhibits the TSC1/TSC2 complex. As a result, the TOR and S6k pathway is not suppressed and cell growth is activated in Drosophila. 
Expression levels of genes thought to be involved in response to stress, nutrients and starvation

\begin{tabular}{|c|c|c|c|c|}
\hline $\mathrm{S} / \mathrm{F}$ & $\mathrm{JH} / \mathrm{S}$ & $\mathrm{E} / \mathrm{F}$ & Name & Biological process affected \\
\hline $1.11(0.036)$ & $0.17(0.042)$ & $-0.77(0.043)$ & to (takeout) & Response to starvation \\
\hline $0.85(0.038)$ & $-1.35(0.003)$ & $-0.15(0.710)$ & bsk (basket) & Response to stress, JNK, MAPK cascade \\
\hline $1.57(0.025)$ & $-1.00(0.007)$ & $1.04(0.020)$ & POSH (Plenty of SH3s) & Receptor signaling complex, scaffold activity \\
\hline $0.04(0.678)$ & $-0.16(0.027)$ & $-0.55(0.038)$ & Tor (Target of rapamycin) & Positive regulation of cell size and cell growth \\
\hline$-0.95(0.004)$ & $1.00(0.007)$ & $-1.45(0.008)$ & Aktl (Protein kinase B) & Protein serine/threonine kinase activity, anti-apoptosis \\
\hline $0.54(0.044)$ & $-0.58(0.042)$ & $-0.07(0.797)$ & Tscl (Triple sex combs 1) & Negative regulation of cell size and cell growth \\
\hline$-0.33(0.002)$ & $0.01(0.423)$ & $0.07(0.094)$ & Dstpk61* & Positive regulation of cell size \\
\hline $0.90(0.013)$ & $0.75(0.043)$ & $-0.14(0.607)$ & Thor (Thor) & Negative regulation of cell size \\
\hline$-0.68(0.021)$ & $-1.18(0.033)$ & $1.14(0.004)$ & $\operatorname{LspI}^{\beta(}\left(\right.$ Larval serum protein $I^{\beta)}$ & Nutrient reservoir \\
\hline$-1.09(0.008)$ & $1.07(0.033)$ & $-0.38(0.038)$ & $\operatorname{LspI}^{\gamma}\left(\right.$ Larval serum protein $\left.I^{\gamma}\right)$ & Nutrient reservoir \\
\hline
\end{tabular}

b

RT-PCR analysis of transcripts from the genes identified by the microarray analysis.

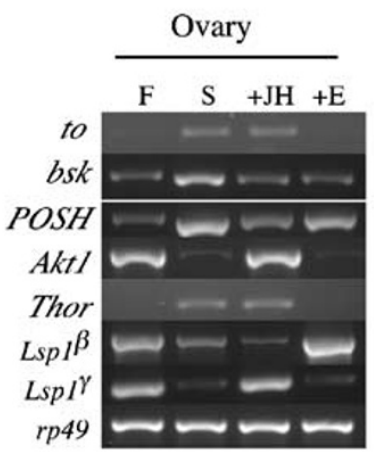

C Gene hierarchy involved in Drosophila body size control.

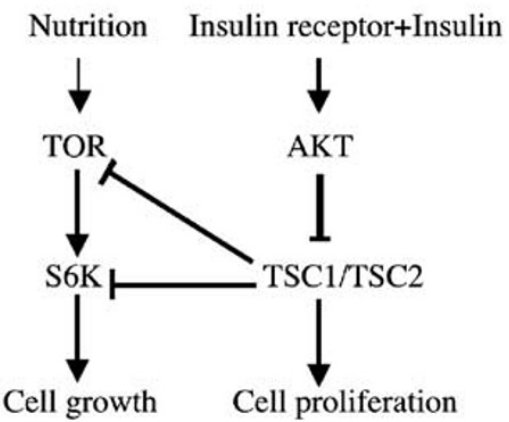

Figure 2 The expression of nutrition- and stress-related genes in the ovary. (a) The nutrition and stress response gene expression levels comparing apoptotic and nonapoptotic conditions is shown. Red - gene expression was upregulated. Yellow - gene expression was not changed. Green - gene expression was downregulated under apoptotic conditions. Starved females were compared with fed females (S/F) and JH-treated females $(\mathrm{S} / \mathrm{JH})$. 3nbh 20E-injected females were compared with fed (E/F) females. The gene names are shown as abbreviations with full names in parentheses. Index $=\log _{2}$ (detected signal ratio) and ()$=P$-value. (b) The RT-PCR analysis. Total RNA was extracted from the ovary containing stages prior to 10 (Ovary) and the egg chambers at stages 8 and 9 (Stages 8 and 9 ). F, S, $+\mathrm{JH}$ and $+\mathrm{E}$ represent fed, starved, JHA treatment and $20 \mathrm{E}$ treatment, respectively. (c) Cell growth and proliferation pathway that is known to be controlled by nutrition and insulin. This scheme is modified from Potter and $\mathrm{Xu}^{17}$

We observed that Akt1, Tsc1, Tor, Thor and Dstpk61 are expressed in the ovary (Figure 2a). The expression of positive cell size regulators, Akt1 and Dstpk61, was downregulated and negative cell size regulators, Tsc1 and Thor were upregulated by starvation (Figure 2a). However, expression of the positive cell size regulator, Tor did not change under starvation. JHA treatment suppressed the expression of Tor and Tsc1, and enhanced the expression of $A k t 1$ and Thor and did not affect Dstpk61 expression. 20E treatment suppressed the expression of Tor and Akt1, but did not affect the expression of Tsc1, Dstpk61 and Thor (Figure 2a). RT-PCR showed the same results for Akt1 and Thor expression (Figure $2 \mathrm{~b}$ ). Expression of the positive cell size regulator, $A k t 1$ was suppressed by starvation and that of the negative cell size regulator, Thor was enhanced by starvation (Figure 2b).

The genes that positively regulate cell and body size namely Akt1 and Dstpk61 were downregulated following starvation. On the other hand, the genes that negatively regulate cell and body size Tsc1 and Thor were upregulated by starvation. These results suggest that cell size in the egg chamber may be downregulated under starvation and upregulated under adequate nutritional conditions.

The cells of the fat body of Drosophila larvae produce large amounts of storage proteins, generally referred to as hexamerins. ${ }^{22}$ Two immunologically distinct hexamerins have been identified as larval serum proteins (LSP). ${ }^{23,24}$ LSP contains LSP1 and LSP2, and LSP1 is composed of three subunits, $\alpha, \beta$ and $\gamma$, each encoded by different genes scattered throughout the genome. ${ }^{24,25}$ Larval serum protein $1 \beta(L s p 1 \beta)$ and $\gamma(L s p 1 \gamma)$ expression in the ovary were downregulated by starvation. $L s p 1 \gamma$ expression was lower under all apoptotic conditions, 20E injection suppressed and JHA treatment enhanced expression. However, $L s p 1 \beta$ expression was suppressed by JHA treatment and $20 \mathrm{E}$ injection induced higher expression in fed flies (Figure 2a and $b$ ).

Thus, we see changes in gene expression within the ovary of genes known to be involved in the stress response or known to be related to the nutritional status of the organism in other tissues or at other times in development. It was not previously known for several of these genes that they were expressed in the ovary. 
a

Expression of hormone related genes

Down-regulated

\begin{tabular}{|c|c|c|}
\hline $\mathrm{S} / \mathrm{F}$ & $\mathrm{JH} / \mathrm{S}$ & $\mathrm{E} / \mathrm{F}$ \\
\hline $0.79(0.009)$ & $0.31(0.017)$ & $-0.58(0.025)$ \\
\hline $1.10(0.023)$ & $-1.00(0.014)$ & $0.92(0.023)$ \\
\hline$-0.03(0.441)$ & $0.77(0.047)$ & $-0.75(0.036)$ \\
\hline$-1.29(0.012)$ & $-0.53(0.048)$ & $0.62(0.009)$ \\
\hline $0.38(0.042)$ & $-0.05(0.184)$ & $-0.11(0.509)$ \\
\hline $0.52(0.009)$ & $-0.39(0.004)$ & $0.71(0.003)$ \\
\hline
\end{tabular}

\begin{tabular}{|l|l|}
\hline Name & Biological process affected \\
\hline dib (disembodied) & Ecdysone biosynthesis, Ecdysteroid 22-dehydrolxyase activity \\
\hline Dare (defective in the avoidance of repellents) & Cholesterol catabolism \\
\hline EcR (Ecdysone receptor) & Ecdysone receptor activity \\
\hline Vha44 (Vacuolar $\mathrm{H}^{+}$ATPase 44kD subunit) & Regulation of JH biosynthesis \\
\hline Jheh2 (Jtvenile hormone epoxide hydrolase2) & $\mathrm{JH}$ catabolism \\
\hline Jheh3 (Juvenile hormone epoxide hydrolase3) & $\mathrm{JH}$ catabolism \\
\hline
\end{tabular}

b

RT-PCR analysis of the transcripts for genes identified by the microarray analysis.
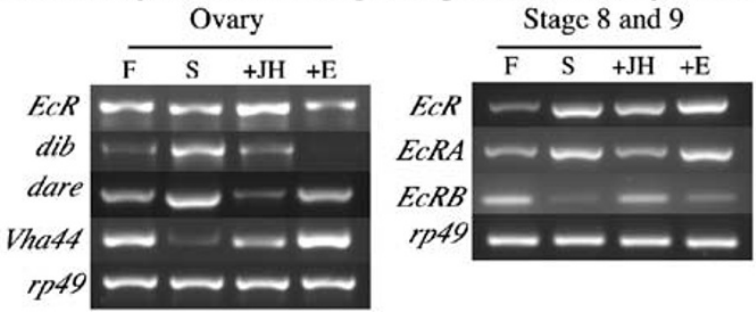

Figure 3 The expression of genes related to hormone biosynthesis and metabolism. (a) The expression of genes that could affect the titre of $\mathrm{JH}$ and ecdysone is shown. Red - gene expression upregulated. Yellow - gene expression was not changed. Green - gene expression was downregulated under apoptotic conditions. Starved flies were compared with fed females (S/F) and JH-treated females $(\mathrm{S} / \mathrm{JH})$. 20E-injected females were compared with fed females $(\mathrm{E} / \mathrm{F})$. Index $=\log _{2}(\mathrm{detected}$ signal ratio) and ()$=P$-value. (b) The RT-PCR analysis. Total RNA was extracted from the ovary containing egg chambers up to stage 10 only and the egg chambers at stages 8 and 9 (Stages 8 and 9). F, S, $+\mathrm{JH}$ and $+\mathrm{E}$ indicate fed, starved, JHA treatment and 20E treatment, respectively

\section{Genes affecting hormone metabolism}

Although it is well documented that $\mathrm{JH}$ and ecdysone modulate the progress of oogenesis, it is not clear how some egg chambers can make a decision to develop while others undergo apoptosis when the haemolymph levels of hormone will be the same for all egg chambers in a given female. Our results show changes in expression of some genes that could well affect metabolism of the $\mathrm{JH}$ and ecdysone within the egg chambers (Figure $3 a$ and $b$ ).

Cholesterol metabolism, essential to produce $20 \mathrm{E}$, is mediated by 22-hydroxylase, 2-hydroxylase and 20-hydroxylase, which are encoded by disembodied (dib), shadow (sad) and shade, respectively. ${ }^{26,27}$ In addition, it is possible that defective in the avoidance of repellents (Dare) interacts to synthesize ecdysteroids. ${ }^{28}$ In our microarray and RT-PCR, dib expression in the ovary of starved flies showed a higher expression than in the ovary of fed flies. JHA treatment induced higher expression and 20E treatment suppressed it (Figure $3 \mathrm{a}$ and b). Dare expression also changed between starvation and feeding conditions. In addition, JHA treatment suppressed its expression (Figure $3 a$ and b). The microarray analysis showed that $20 \mathrm{E}$ treatment induced dare expression, but the results of RT-PCR did not show much difference in dare expression between fed and $20 \mathrm{E}$ treated females (Figure $3 b$ ). These results suggest that the ecdysone synthesis pathway is activated in the ovary itself by starvation and includes the expression of dib and Dare.

We have shown that some ecdysone response genes induce apoptosis of egg chambers at stages 8 and 9 of oogenesis in Drosophila. ${ }^{3}$ Under apoptotic conditions, E75A, $B R-C Z 2$ and $Z 3$ are expressed in egg chambers at stages 8 and 9 (Terashima and Bownes, ${ }^{3}$ and unpublished data); this results in the induction of apoptosis in egg chambers. In addition, ecdysone response genes and ecdysone receptor (EcR) need to be expressed for normal oogenesis to proceed. In clones of cells mutant for $E 75$ and $E c R$, oogenesis is arrested at mid-oogenesis stages and egg chambers degenerate. ${ }^{29,30} E c R$ expression showed no difference between fed and starved ovaries in the microarray analysis and RT-PCR, but JHA treatment induced and $20 \mathrm{E}$ treatment suppressed expression (Figure $3 \mathrm{a}$ and $\mathrm{b}$ ). We thought that the $E c R$ expression in stage 8 and 9 egg chambers could be important for inducing apoptosis. Therefore, we checked the expression of $E c R$ isoforms, $E c R A$ and $E c R B$, in egg chambers at stages 8 and 9 by RT-PCR (Figure 3b). EcR expression at stages 8 and 9 was enhanced by starvation and $20 \mathrm{E}$ treatment, and JHA treatment suppressed this expression (Figure $3 \mathrm{~b}$ ). This result was mirrored by the expression of EcRA at stage 8 and 9, EcRA expression was enhanced by starvation and $20 \mathrm{E}$ treatment but suppressed by JHA treatment. However, EcRB expression at stages 8 and 9 showed the opposite expression pattern being suppressed by starvation and $20 \mathrm{E}$ treatment and enhanced by JHA treatment (Figure $3 b$ ). These results suggested that EcRA and $B$ may have opposite affects in relation to inducing apoptosis of stage 8 and 9 egg chambers. $E c R A$ may induce expression of $E 75 A, B R-C Z 2$ and $Z 3$, all apoptosis inducers by binding ecdysone and ultraspiracle.

$\mathrm{JH}$ epoxide hydrolase (JHEH) may play a pivotal role in regulating insect juvenile hormone $(\mathrm{JH})$ titers along with $\mathrm{JH}$ esterase. ${ }^{31}$ Vacuolar $H^{+}$ATPase $44 \mathrm{kDa}$ subunit (Vha44) may also be involved in $\mathrm{JH}$ synthesis. ${ }^{32}$ Under starvation conditions, Juvenile hormone epoxide hydrolase 2 (Jheh2) and 3 gene expression were upregulated and Vha44 expression was downregulated (Figure 3a). 
Further investigations of the functions of these genes in the ovary and how titers of hormones may change within the ovary itself would be a good way forward to investigate exactly how the regulation of hormone titers may affect and be affected by the ovary. It also may explain how different egg chambers in the same haemolymph can respond differently to the environmental conditions present.

\section{Apoptosis-related genes}

Since the end result of nutritional deprivation is apoptosis of many egg chambers, it is not surprising that expression of many genes known to be involved in apoptosis at other stages of the life cycle are expressed at higher levels in the ovaries of starved flies than fed flies.

$D P$ transcription factor $(D p)$ is a positive regulator of nurse cell apoptosis and interacts with E2F, a transcription factor (E2f). ${ }^{33}$ The Drosophila E2F includes E2F and E2F transcription factor 2 (E2f2). ${ }^{33} D p$ was upregulated in starved flies and was suppressed by JHA treatment in the ovary containing egg chambers up to stage 9 and the egg chambers at stages 8 and 9 (Figure 4a and b). 20E treatment did not affect $D p$ expression in the ovary excluding all egg chambers beyond stage 10 (Figure 4a), but in the egg chambers at stages 8 and 9, Dp expression was enhanced by $20 \mathrm{E}$ treatment (Figure $4 \mathrm{~b}$ ). Dp expression was detected in the nurse cells and oocytes of egg chamber at stage 9 under all conditions. Expression at stage 8 was weaker than at stage 8 following feeding and JHA treatment. The expression at stage 8 during starvation and $20 \mathrm{E}$ treatment was stronger than during feeding and JHA treatment (Figure 5a). E2f2 was upregulated in the ovary of starved flies in a similar way to $D p$ expression. However, JHA enhanced E2f2 expression in starved flies and $20 E$ suppressed expression in the fed flies. licorne (lic) is a MAPK kinase, targeting the MAPK known as $p 38 .^{34}$ It is known that p38 signalling has a role in apoptosis and is activated by many environmental stress signals. ${ }^{35}$ lic expression was enhanced by starvation and 20E treatment, and JHA did not affect its expression (Figure 4a).

The caspase pathway is one of the important pathways for inducing apoptosis in Drosophila (reviewed in Meier et al. ${ }^{36}$ ). Nedd 2-like caspase (Nc, Dronc) and dream have caspase activity. ${ }^{37,38} \mathrm{Nc}$ (DRONC) was upregulated by starvation and $20 \mathrm{E}$ treatment of fed flies, but there are no differences in the expression levels between the ovary of starved flies and JHAtreated flies in the ovary containing stages up to stage 10 (Figure 4a). Nc expression in the egg chambers at stages 8 and 9 was also enhanced by starvation and $20 \mathrm{E}$ treatment; moreover, JHA treatment suppressed Nc expression under starvation (Figure 4b). Nc expression was detected in the nurse cells of stage 8 and 9 egg chambers during starvation and $20 \mathrm{E}$ treatment (Figure 5a), but expression was not

a

Expression of apoptosis related genes

Up-regulated

No-change

Down-regulated

\begin{tabular}{|c|c|c|}
\hline $\mathrm{S} / \mathrm{F}$ & $\mathrm{JH} / \mathrm{S}$ & $\mathrm{E} / \mathrm{F}$ \\
\hline $0.99(0.027)$ & $-0.87(0.012)$ & $-0.07(0.670)$ \\
\hline $0.65(0.045)$ & $0.52(0.021)$ & $-0.91(0.021)$ \\
\hline $0.59(0.034)$ & $-0.05(0.857)$ & $1.27(0.048)$ \\
\hline $1.35(0.038)$ & $-1.04(0.0002)$ & $1.24(0.047)$ \\
\hline $0.96(0.001)$ & $-2.00(0.024)$ & $0.64(0.002)$ \\
\hline $0.99(0.034)$ & $-0.58(0.025)$ & $0.67(0.017)$ \\
\hline $0.80(0.043)$ & $-0.04(0.888)$ & $0.70(0.038)$ \\
\hline$-0.03(0.675)$ & $1.04(0.024)$ & $-1.00(0.036)$ \\
\hline$-0.60(0.045)$ & $0.05(0.891)$ & $-0.05(0.734)$ \\
\hline$-1.86(0.040)$ & $0.50(0.026)$ & $-0.80(0.041)$ \\
\hline
\end{tabular}

\begin{tabular}{|l|l|}
\hline Name & \multicolumn{1}{|c|}{ Biological process affected } \\
\hline$D p$ (DP transcription factor) & Positive regulation of nurse cell apoptosis \\
\hline$E 2 f$ (E2F transcription factor 2) & Interaction with E2F (Positive regulation of nurse cell apoptosis) \\
\hline$N c$ (Nedd 2-like caspase) & Caspase activity \\
\hline dream (dream) & Apoptosis inducer activity \\
\hline$p 53$ (p53) & Apoptosis inducer activity, response to radiation \\
\hline lok (loki) & DNA damage response, resulting in induction apoptosis \\
\hline lic (licorne) & MAPKK activity \\
\hline th (thread) & IAP1 activity, apoptosis inhibitor activity \\
\hline lap2 (inhibitor protein of apoptosis 2) & Apoptosis inhibitor activity \\
\hline Aac11 (Aac11) & Apoptosis inhibitor activity \\
\hline
\end{tabular}

b

RT-PCR analysis of the transcripts for genes identified by the microarray analysis.

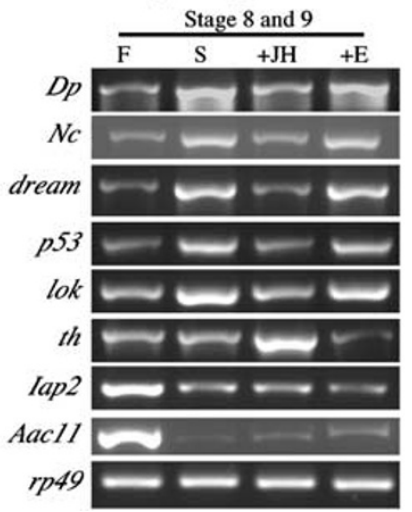

Figure 4 The expression of apoptosis-related genes. (a) Apoptosis-related gene expression levels. Red - gene expression was upregulated. Yellow - gene expression was not changed. Green - expression was downregulated under apoptotic conditions. Starved flies were compared with fed females (S/F) and JH-treated females (S/ $\mathrm{JH})$. 20E-injected females were compared with fed females (E/F). Index $=\log _{2}$ (detected signal ratio) and ()$=P$-value. (b) The RT-PCR analysis. Total RNA was extracted from the egg chambers at stages 8 and 9 (Stages 8 and 9). F, S, + JH and $+E$ indicate fed, starved, JHA treatment and 20E treatment, respectively 
A

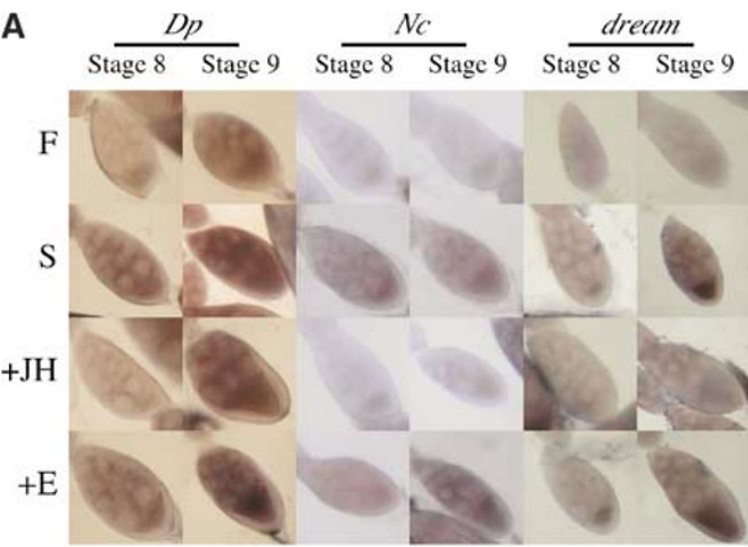

B

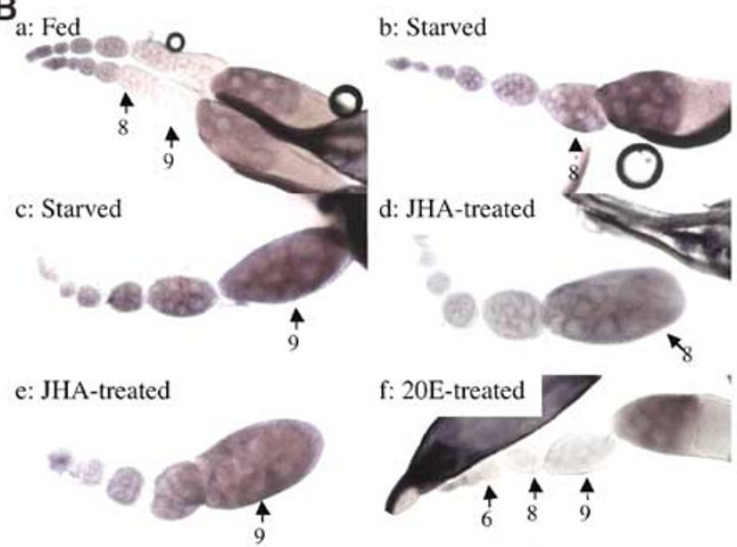

Figure 5 Temporal and spatial expression of apoptosis-related genes in oogenesis. (A) The expression patterns of $D p, N c$ and dream at stage 8 and 9 egg chamber, as detected by in situ hybridization. $\mathrm{F}, \mathrm{S},+\mathrm{JH}$ and $+\mathrm{E}$ indicate fed, starved, JHA treatment and 20E treatment, respectively. (B) th expression in the ovary: a, the ovary from fed female, $b$ and c, starved females; $d$ and e, JHAtreated females and $\mathrm{f}, 20 \mathrm{E}$-treated females. The numbers on the panels indicate stages

detected in the ovaries of fed and JHA-treated females. dream was upregulated under starvation and following $20 \mathrm{E}$ treatment and suppressed by $\mathrm{JHA}$ treatment. $p 53$, crucial for inducing apoptosis, responds to DNA damage and reaper is a direct transcriptional target of Drosophila p53 following DNA damage ${ }^{39}$ however, $p 53$ has not reported as an inducer of developmental apoptosis in Drosophila and its function in oogenesis is unknown. loki (lok) is the Drosophila chk-homolog and lok regulates irradiation-induced, p53mediated apoptosis in Drosophila. ${ }^{40}$ p53 and lok were upregulated under all apoptotic conditions (starvation and $20 \mathrm{E}$ treatment) in the ovary containing only egg chambers prior to stage 10 and the egg chambers at stages 8 and 9 (Figure $4 a$ and b). This means that p53 and lok were induced by starvation and $20 \mathrm{E}$ treatment and suppressed by JHA application.

The Drosophila homologue of inhibitor of apoptosis 1 (lap1) is thread (th) and Drosophila also have the inhibitor of apoptosis 2 (lap2).$^{41}$ Starvation did not suppress th expression, but $20 \mathrm{E}$ treatment suppressed and JHA treatment enhanced it. lap2 expression was suppressed by starvation and $20 \mathrm{E}$ treatment, but there was no change comparing starvation and JHA treatment (Figure 4a). th and lap2 expression in the egg chamber at stages 8 and 9 showed same results as the microarray (Figure $4 b$ ).

Aac11 has apoptosis inhibitor activity ${ }^{42}$ and its expression was suppressed by starvation and $20 \mathrm{E}$ treatment in the ovary containing egg chambers up to stage 10 and the stage 8 and 9 egg chambers; and JHA treatment induced expression in the ovary excluding stages beyond stage 10 (Figure $4 a$ and $b$ ). However, in the egg chambers at stages 8 and 9, JHA treatment had no effect Aac11 expression (Figure 4b).

Figure $5 \mathrm{~b}$ shows th expression in the ovary. th expression was not detected in the stage 8 and 9 egg chambers and $20 \mathrm{E}$ treatment suppressed th expression almost completely, except in the nurse cells at stage 10 (Figure 5Ba) Under starvation and $\mathrm{JHA}$ treatment, th was expressed in many egg chambers at stages 8 and 9 (Figure $5 \mathrm{Bb}-\mathrm{d}$ ).

Some apoptosis inducer gene expression showed different expression patterns in the egg chambers at stages 8 and 9 when comparing adequate nutrition, JHA treatment and starvation and $20 \mathrm{E}$ treatment. We propose that these different expression patterns are important for inducing apoptosis in the egg chamber at stages 8 and 9. One of the apoptosis inhibitors, th, is expressed in many egg chambers at stages 8 and 9 under starvation, but is not expressed under adequate nutrition. We suggest that th is expressed in those egg chambers at stages 8 and 9 that survive and develop to mature eggs under starvation and that it acts as an apoptosis inhibitor.

\section{Discussion}

\section{Nutrient and stress response pathways in the ovary}

The genes we see with changing expression levels in the microarray experiment support the model shown in Figure 6, suggesting how the ovary responds to environmental change. Red and Green indicate the genes that are down and upregulated by nutritional shortage and/or 20E treatment. Nutrient shortage is one of several environmental stresses for Drosophila. Probably, many tissues and organs respond to nutritional shortage; however, until these experiments were undertaken we did not know how the ovary responded. The ovary is affected by nutritional shortage by ultimately reducing yolk protein synthesis, by fewer eggs being laid and we know there is an altered expression pattern of some ecdysone response genes. ${ }^{2,3}$ We propose that the transmission of the nutritional response signal includes stress responses. It is likely that the nutrient and stress response pathways in other tissues, such as the gut and fat body, respond to nutrient shortage, and that signals from these tissues activate a subsequent pathway in the ovary. It seems, however, that regulation of hormone concentration occurs within the ovary itself and this, one might have expected, would be controlled entirely by other tissues.

The gut is one of the candidates for releasing the first signal to respond to nutritional status. It is possible that the gut monitors the intake of the fly and releases signals into the haemolymph. A midgut hormone in liver-fed Phormia regina adult females leads to the completion of oogenesis. ${ }^{43}$ 


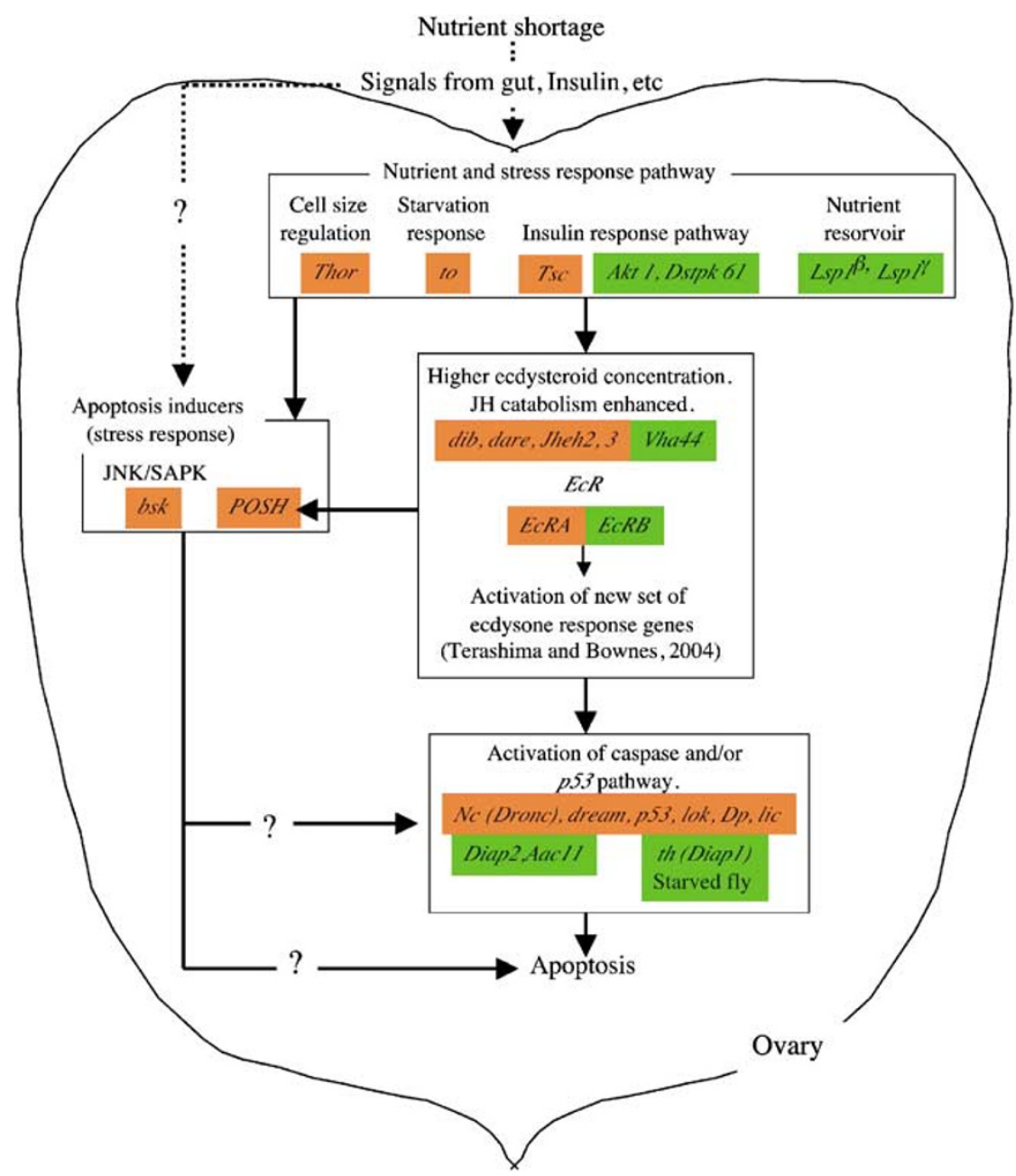

Figure 6 Model linking nutritional status and apoptosis in the egg chamber. Nutrient shortage activates or inactivates the nutrient and stress response pathway directly or indirectly. It is possible that genes that modulate ecdysone and $\mathrm{JH}$ levels are downstream of the nutrient and stress response pathway. Ecdysone activates a new set of ecdysone response genes, and these genes activate the caspase pathway or p53 pathway to induce apoptosis. It is possible that there is JNK/SAPK pathway downstream of stress response pathway in the ovary. In this case, the JNK/SAPK pathway would need to be affected by ecdysone and ecdysone response genes, because to induce the apoptosis of egg chamber at stages 8 and 9 needs higher ecdysone concentration and ecdysone response gene expression is enhanced. The apoptosis of egg chambers at stages 8 and 9 of oogenesis has three possible apoptosis pathways, the JNK/SAPK pathway, the caspase pathway and the p53 pathway. It is likely that these three pathways interact each other and induce the apoptosis observed at stages 8 and 9

Subsequently, the nutrient and stress response pathways in the ovary respond themselves to nutritional shortage, since the expression of some nutrient and stress response genes are up- or downregulated within the ovary (Figure $2 a$ and b). The nutrient and stress response genes are categorized into subgroups (Figure 6). We expect that insulin has an important role for sensing nutritional status, and insulin release from the gut should be reduced under nutritional shortage. The ovary of insulin receptor mutants are immature and arrest at midoogenesis, ${ }^{44}$ and chico mediates insulin receptor signalling in Drosophila, and it has a significant effect on egg chamber progression into vitellogenesis. ${ }^{1}$ We propose that the insulin response pathway is inactivated by lack of insulin from the gut and suppresses cell growth (Figures $2 c$ and 6). In the fed fly, the insulin receptor in the ovary activates AKT and induces cell growth. On the other hand, in starved flies, the insulin receptor is inactivated resulting in inactivation of AKT. This inactivation could lead to the activation of the TSC1/TSC2 complex and induce cell proliferation. In this case, gut or other tissues would release signals like insulin to activate or inactivate the nutrition and stress response pathway in the ovary. We suggest that the ovary then uses the genes which show altered expression levels to control hormone concentration and ultimately, therefore, the apoptosis pathway. The insulin receptor interacts with AKT and AKT interacts with DSTPK61. ${ }^{21}$ The Akt1 product has a role in antiapoptosis, ${ }^{45}$ and the Dsptk61 product is a positive regulator of cell size. ${ }^{21,46}$ The expression of both these genes in the ovary were downregulated by starvation. In addition, Tsc1 and Thor, which are negative regulators of cell size, were upregulated by starvation (Figure 2a and b). Nutritional shortage signals should modulate cell size and nutrient storage suppression and prepare cells to induce apoptosis in the egg chamber at stages 8 and 9 . 


\section{How might apoptosis be induced?}

It seems likely that nutrition and stress response pathways regulate ecdysone concentration in the ovary. The ovary is one of several tissues in the adult that is capable of ecdysone synthesis, ${ }^{47,48}$ and it is possible that secretion of ecdysone from ovary itself can affect ecdysone concentration in the haemolymph. Simultaneously, other tissues that synthesize ecdysone may also affect the concentration in the fly, and it has been shown that the total ecdysone concentration in the fly is increased in starved flies. ${ }^{10}$ In the ovary, dib and/or Dare expression could affect the synthesis of ecdysone or $20 \mathrm{E}$. Higher ecdysone concentration in the fly induces apoptosis of the egg chamber at stages 8 and $9,,^{2,3}$ and overexpression of some ecdysone response genes induce apoptosis of egg chambers at stages 8 and $9 .^{3}$ Therefore, we expect that to induce the apoptosis at stages 8 and 9 requires an increase in ecdysone concentration and either a decrease in $\mathrm{JH}$ concentration or the suppression of $\mathrm{JH}$ action. Vha44, a JH synthesis-related gene is suppressed and $\mathrm{JH}$ catabolism genes, Jheh2 and 3 are enhanced by starvation. This hormone-related gene expressions is probably controlled by the nutrient and stress response pathways, and as discussed in the previous section, the strongest candidate is the insulin pathway. Insulin receptor mutant females require continuous exposure to JHA to induce any vitellogenesis. ${ }^{45}$

The EcR with ecdysone bound to it and Ultraspiracle act as a transcription factor. Oogenesis in $E c R$ mutant is arrested at mid-oogenesis and the egg chambers are degenerated. ${ }^{29}$ This means that the EcR is essential to progress normal oogenesis. However, the results of RT-PCR suggest EcR may also act in the egg chamber at stages 8 and 9 to induce the apoptosis of the nurse cells. EcRA expression in the egg chamber at stages 8 and 9 was enhanced by starvation and $20 \mathrm{E}$ treatment (Figure $3 \mathrm{~b}$ ) but EcRB expression in the egg chamber at stages 8 and 9 was suppressed by starvation and $20 \mathrm{E}$ treatment. These results suggest that $E c R A$ acts as transcription factor for the genes involved in inducing apoptosis such as $B R-C Z 2$ and $Z 3$. $^{3}$ We predict that EcR should have affects not only on normal oogenesis but also on the induction of apoptosis, possibly different isoform of $E c R$ have different roles in normal oogenesis and in the induction of apoptosis during Drosophila oogenesis.

There are some candidate genes for the pathway that leads to apoptosis. $D p$, lic, $p 53$, JNK/SAPK and the caspase family were identified by the microarray, RT-PCR and in situ hybridization. $D p$ normally induces the apoptosis of nurse cells that commences at stage 10 during normal oogenesis and is known to interact with E2fand E2f2. ${ }^{34}$ Most apoptosis in Drosophila is induced by the caspase, including Nc (Dronc) and dream, pathway that is activated by ecdysone response genes. ${ }^{37}$ The germ line of nutrient-deprived flies carrying either a loss of function mutation in Death caspase-1 (Dcp-1) or a transgene overexpressing the caspase inhibitor Diap1 fail to die, and large numbers of abnormal egg chambers accumulate in the ovaries. ${ }^{49,50}$ In our microarray analysis, Dcp-1 expression was increased by nutrient shortage and $20 \mathrm{E}$ treatment and suppressed by JHA treatment (Index, $\mathrm{S} / \mathrm{F}=1.00, \mathrm{JH} / \mathrm{S}=-0.95, \mathrm{E} / \mathrm{F}=0.89)$, but all the $P$-values were over 0.05 (Data not shown) and therefore was not significant and could not be included in our list of genes altered by these experimental conditions. Nonetheless, we expect that $D c p-1$ is likely to interact with the apoptosis process during mid-oogenesis. The apoptosis activity of p53 is regulated by lok in Drosophila. ${ }^{41}$ lic is the homologue of $p 38$ in Drosophila and is required for oogenesis. ${ }^{35} \mathrm{POSH}$ belongs to the JNK/SAPK pathway downstream of the stress response pathway and induces apoptosis in the Drosophila wing and neural tissues. ${ }^{14-16}$ These genes are regulated by $20 \mathrm{E}$, probably mediated by $B R-C Z 2, Z 3$ and $E 75 A$. bsk also belongs to JNK/SAPK pathway although starvation induced bsk expression, 20E treatment did not induce its expression (Figure $2 \mathrm{a}$ and $\mathrm{b}$ ). This suggests that there is one ecdysonedependent pathway and another ecdysone-independent pathway that can induce apoptosis. $20 \mathrm{E}$ treatment of fed females induces ovarian apoptosis ${ }^{2,3}$ and this requires changes in expression of response genes. It seems likely that an increase in ecdysone concentration in the fly is essential for inducing the apoptosis in the egg chamber at stages 8 and 9 . We propose that bsk may support the main apoptosis pathway. In addition, there are some candidates for apoptosis inhibitors, including thread, lap2 and Aac11. The homologues of the apoptosis inhibitors, lap1 and 2 have been identified as thread (th, Drosophila lap1, Diap1) and Drosophila lap2 (Diap2). ${ }^{41}$ Aac11 has apoptosis inhibitor activity in mammalian cells, ${ }^{42}$ but the mechanism of action is unknown. th expression levels did not change between the ovary of starved flies and fed flies. However, we have observed that $t h$ expression at stages 8 and 9 differed spatially and temporally between the ovaries of starved flies and fed flies (Terashima and Bownes, unpublished data). th was not expressed in the egg chambers at stages 8 and 9 in fed flies, but was expressed in some egg chambers in starved flies. This expression pattern has been reported by Foley and Cooley. ${ }^{5}$ They have shown the Diap1 expression is detected beginning at region $2 \mathrm{~B}$ in the germarium and downregulated at mid-oogenesis stages. ${ }^{5}$ In addition, as we have mentioned, flies overexpressing the caspase inhibitor Diap1 as a transgene fail to die and large numbers of abnormal egg chambers accumulate in the ovaries. ${ }^{50}$ We expect that $t h$ is expressed in the small number of egg chambers of starved flies that survive and develop into mature eggs. In starved flies, some egg chambers always show normal development ${ }^{3}$ and we suggest that th has a role inhibiting the apoptosis at stages 8 and 9 of these egg chambers. Diap 2 expression is detected in the nurse cells of these egg chambers. ${ }^{5}$ Although lap2 expression was suppressed by starvation, JHA treatment did not affect its expression in the ovary of starved flies. Perhaps th and lap2 inhibit apoptosis in starved flies and fed flies, respectively. Probably, starvation signals switch the affects of the apoptosis inhibitors th and lap2 in early oogenesis.

We propose a model for inducing apoptosis in egg chambers at stages 8 and 9 by starvation. The genes that are candidates for inducing the apoptosis are grouped as nutrient and stress response, hormone-related and apoptosisrelated genes. The nutrient and stress response group includes the genes that are related to JNK/SAPK, and the insulin pathway that control cell size regulation and nutrient storage. Some of these genes should regulate hormone synthesis or catabolism to increase ecdysone concentration. 
The increase in ecdysone levels must activate the apoptosis executing pathway, caspase, $p 53$ and/or JNK/SAPK. One of the JNK/SAPK genes $b s k$ may act as an apoptosis inducer that is independent of ecdysone concentration or has other unknown affects in oogenesis. We predict that lack of an insulin signal marks beginning of activation of the apoptosis pathway in the mid-oogenesis, and probably induces ecdysteroid synthesis. Tu et al. ${ }^{51}$ reported insulinlike receptor mutant virgin females show reduced production of ecdysteroids in the ovary. In our measurement, ecdysteroid secretion from the ovaries was decreased, but the ecdysteroid concentration in the ovaries was increased in Drosophila after mating (Terashima J, Takaki K, Sakurai S, and Bownes M, unpublished data). We therefore predict that the higher ecdysteroid concentration may be induced by lack of an insulin signal. In this paper, we have identified some of the candidate genes in the ovary for sensing nutrient changes and stress, modulating hormone titre and initiating and executing apoptosis.

\section{Materials and Methods}

\section{Drosophila maintenance}

Flies were maintained on standard yeast, maize meal, sugar and agar medium at $25^{\circ} \mathrm{C}$. The wild-type strain, Oregon $R$ was used throughout. Flies (3-day old) were transferred from a standard diet to one of sugar and water (starved: $1 \%$ agar medium that contains $5 \%$ sucrose and $0.005 \%$ Nipagen in $95 \%$ ethanol) or one of yeast (fed: $2 \mathrm{~g}$ bakers yeast on approximately $50 \mathrm{ml} \mathrm{1 \%}$ agar medium that contains $2.5 \%$ cornflour, $5 \%$ sucrose, $1.75 \%$ lyophilized yeast and $0.005 \%$ Nipagen in $95 \%$ ethanol). After 3 days on sugar or yeast, flies were dissected (Sugar: S3, Yeast: F3), transferred to sugar for 1 day after 3 days on yeast (F3S1), or topically treated with Methoprene and maintained on sugar and water for 1 day (F3JHS1), or injected with 20E and maintained on yeast for 1 day (F3EF1).

\section{Injection of 20E and application of JHA}

$20 \mathrm{E}$ (Sigma) was dissolved in Insect Ringer's solution $(130 \mathrm{mM} \mathrm{NaCl}$, $4.7 \mathrm{mM} \mathrm{KCl}, 1.9 \mathrm{mM} \mathrm{CaCl}_{2}$ ) and $50 \mathrm{nl}$ was injected at a concentration of $2 \mu \mathrm{g} / \mathrm{ml} .{ }^{52}$ The concentration of $20 \mathrm{E}$ was determined according to Bownes, ${ }^{10}$ with a haemolymph volume of approximately $1 \mu / /$ female, ${ }^{52}$ injecting $100 \mathrm{pg} 20 \mathrm{E} / \mathrm{female}$ leads to a concentration of $2 \times 10^{-7} \mathrm{M}$. Methoprene (ZR515, Zoecon) was applied topically to the ventral abdomen in $100 \mathrm{nl}$ acetone. Methoprene diluted 1:100 in acetone corresponds to a concentration of about $1 \mu \mathrm{g} / 100 \mathrm{nl}$. Controls were undertaken by injecting Ringer's only and treating flies with acetone.

\section{Microarray and data analysis}

The comparisons between experimental conditions in the microarray experiments were starved versus fed, starved versus JHA-treated and $20 \mathrm{E}$-injected versus fed (each three sets). The ovaries were dissected in Insect Ringer's solution and homogenized in $500 \mu \mathrm{l}$ TRIZOL. There are $27-55$ pairs of ovaries in each sample $(40-55 \mathrm{mg})$. The samples were stored at $-80^{\circ} \mathrm{C}$ until undertaking the microarray analysis. When the microarray was carried out, the same volume RNA was used from each sample. There were 5364 genes on the chip and the microarray processes are described on http://www.flychip.org.uk/Project/Protocols.

We prepared three experimental sets for each combination and carried out the microarray. Cy3 (red) and Cy5 (yellow) fluorescence intensities were normalized and nonbackground subtracted. The mean ratios (Cy3 and Cy5) are calculated and sorted by the absolute log ratio. Upregulation or downregulation is determined by the average log ratio (ALR). Upregulation is ALR $>0$ and downregulation is ALR $<0$. The validity of the results is provided by the $P$-value of ALR, which is shown as a percentage. The genes whose $P$-value of the log ratio are over $95 \%$ are categorized as 'no-change' in gene expression and the genes with expression levels that have a significant difference between the test sample and control sample $(P<0.05)$ are either 'up or downregulated'. The expression index was calculated as follows: $\log _{2}$ (the ratio of detected signals, $\mathrm{S} / \mathrm{F}, \mathrm{JH} / \mathrm{S}$ and $\mathrm{E} / \mathrm{F}$ ), as the signals detected by a factor of 2 change have a signal ratio of 2 , whereas the signals detected by the same factor have a signal ratio of -0.5 . The most widely used alternative transformation of the ratio is the logarithm base 2, which has the advantage of producing a continuous spectrum of values and treating upand downregulated genes in a similar fashion. ${ }^{53}$ It is important to remember that logarithms treat numbers and their reciprocals symmetrically: $\log _{2}(1)=0, \log _{2}(2)=1 \log _{2}(1 / 2)=-1, \log _{2}(4)=2, \log _{2}(1 / 4)$ $=-2$, and so on.

\section{In situ hybridization and RT-PCR}

The protocol is based on the procedure previously described ${ }^{54}$ and modified as follows. The ovaries were dissected in Ringer's solution and fixed for $20 \mathrm{~min}$ in $4 \% \mathrm{p}$-formaldehyde in PBS. After rinsing the tissue in PBT, it was treated for $10 \mathrm{~min}$ in methanol/0.5 M EGTA, pH 8 (9:1). The ovaries can then be stored in methanol at $-20^{\circ} \mathrm{C}$ for several months. The stored ovaries were rehydrated in PBT. The prehybridization was carried out for $1 \mathrm{~h}$ at $45^{\circ} \mathrm{C}$ in DNA hybrix ( $50 \%$ deionized formamide, $5 \times \mathrm{SSC}$, $100 \mu \mathrm{g} / \mathrm{ml}$ sonicated salmon sperm DNA, $50 \mu \mathrm{g} / \mathrm{ml}$ Heparin, $0.1 \%$ Tween 20). The ovaries were hybridized overnight at $45^{\circ} \mathrm{C}$ in DNA hybrix containing digoxigenin-labelled probe (DIG-DNA labelling and detection kit, Boehringer Mannheim). For detection, a 1:1000 dilution of anti-DIG$A P$-conjugated $A b$ was used. The staining reaction was performed in $100 \mathrm{mM}$ Tris pH 9.5, $50 \mathrm{mM} \mathrm{MgCl}, 10 \mathrm{mM} \mathrm{NaCl}, 0.2 \%$ Tween $20,8 \mathrm{mM}$ levamisole, $4.5 \mu \mathrm{l} / \mathrm{ml} \mathrm{NBT}$, and $3.5 \mu \mathrm{l} / \mathrm{ml} \mathrm{X-phosphate} \mathrm{(Boehringer}$ Mannheim). Anti-DIG-AP conjugate was preabsorbed with postfixed wild-type (Oregon $\mathrm{R}$ ) ovaries at $4^{\circ} \mathrm{C}$ overnight. The ovaries were mounted in a mixture of PBS/glycerol $(1: 4)$ for microscopy. Egg chambers were staged according to their size and morphology. ${ }^{55}$

The levels of the gene transcripts in ovaries were detected by reverse transcriptase (RT)-PCR as described previously. ${ }^{56}$ The egg chambers after stage 10 were removed from the ovaries and the egg chambers at stages 8 and 9 were isolated from the ovaries in ice-cold Ringer's solution. The details of total RNA extraction, reverse transcription reactions and the PCR reaction are described in Tzolovsky et al. ${ }^{57}$

We used two types of starved flies, S3 (as a control for comparison with F3) and F3S1 (as a control for comparison with F3JHS1) in the microarray analysis. The gene expression levels and the spatial and temporal expression patterns resulting from in situ hybridization were similar between S3 and F3S1 females. Therefore, we show the results of the ovaries in S3 flies as the example for starved flies in the RT-PCR and in situ hybridization analysis.

\section{Acknowledgements}

This Research was supported by a BBSRC project grant and the BBSRC funded microarray facilities in Cambridge and Glasgow. We thank Dr Lisa Meadows in Cambridge and Dr Jing Wang in Glasgow for their support 
with the microarrays, and Hilary Anderson for assistance with preparing the manuscript.

\section{References}

1. Drummond-Barbosa D and Spradling AC (2001) Stem cells and their progeny respond to nutritional changes during Drosohila oogenesis. Dev. Biol. 231: $265-278$

2. Soller M, Bownes M and Kubli E (1999) Control of oocyte maturation in sexually mature Drosophila females. Dev. Biol. 208: 337-351

3. Terashima $J$ and Bownes $M(2004)$ Translating available food into the number of eggs laid by Drosophila melanogaster. Genetics 167: 1711-1719

4. Bownes $M$ and Reid $G$ (1990) The role of ovary and nutritional signals in the regulation of fat body yolk protein gene expression in Drosophila melanogaster. J. Insect. Physiol. 36: 471-479

5. Foley K and Cooley L (1998) Apoptosis in late stage Drosophila nurse cells does not require genes within the H99 deficiency. Development 125 1075-1082

6. White $\mathrm{K}$, Grether ME, Abrams JM, Young L, Farrell $\mathrm{K}$ and Steller $\mathrm{H}$ (1994) Genetics control of programmed cell death in Drosophila. Science 264: 677-683

7. Grether ME, Abrams JM, Agapite J, White K and Steller H (1995) The head involution defective gene of Drosophila melanogaster functions in programmed cell death. Genes Dev. 9: 1694-1708

8. Chen P, Nordstrom W, Gish B and Abrams JM (1996) Grim, a novel cell death gene in Drosophila. Genes Dev. 10: 1773-1782

9. Wilson TG (1982) A correlation between juvenile hormone deficiency and vitellogenic oocyte degeneration in Drosophila melanogaster. Wilhelm Roux's Arch. 191: 251-263

10. Bownes $M$ (1989) The roles of juvenile hormone, ecdysone and the ovary in the control of Drosophila vitellogenesis. J. Insect Physiol. 35: 409-413

11. DiBello PR, Withers DA, Bayer CA, Fristrom JW and Guild GM (1991) The Drosophila Broad-Complex encodes a family of related proteins containing zinc fingers. Genetics 129: 385-397

12. McCall K (2004) Eggs over easy: cell death in the Drosophila ovary. Dev. Biol. 274: 3-14

13. Sarov-Blat L, So WV, Liu L and Rosbash M (2000) The Drosophila takeout gene is a novel molecular link between circadian rhythms and feeding behavior. Cell 101: 647-656

14. Goberdhan DCl and Wilson C (1998) JNK cytoskeletal regulator and stress response kinase? A Drosophila perspective BioEssay 20: 1009-1019

15. Adachi-Yamada T, Fujimura-Kamada K, Nishida $Y$ and Matsumoto K (1999) Distortion of proximodistal information causes JNK-dependent apoptosis in Drosophila wing. Nature 400: 166-169

16. Xu Z, Kukekov NV and Greene LA (2003) POSH acts as a scaffold for a multiprotein complex that mediates JNK activation in apoptosis. EMBO J. 22: 252-261

17. Potter CJ and Xu T (2001) Mechanisms of size control. Curr. Opin. Gen. Dev. 11: $279-286$

18. Oldham $S$ and Hafen $E$ (2003) Insulin/IGF and target of rapamycin signaling: a TOR de force in growth control. Trends cell Biol. 12: 79-85

19. Bernal A and Kimbrell D (2000) Drosophila Thor participates in host immune defense and connects a translational regulator with innate immunity. Proc. Natl. Acad. Sci. USA 97: 6019-6024

20. Kozma SC and Thomas $G$ (2002) Regulation of cell size in growth development and human disease: PI3K PKB and S6K. BioEssays 24: 65-71

21. Alessi DR, Deak M, Casamayor A, Caudwell FB, Morrice N, Norman DG, Gaffrey P, Reese CB, MacDougall CN, Harbison D, Ashworth A and Bownes M (1997) 3-Phosphoinositide-dependent protein kinase-1 (PDK1): structural and functional homology with the Drosophila DSTPK61 kinase. Curr. Biol. 1: 776-789

22. Telfer WH and Kunkel JG (1991) The function and evolution of insect storage hexamers. Annu. Rev. Entomol. 36: 205-228

23. Roberts DB and Evans-Roberts S (1979) The genetic and cytogenetic localization of the three structural genes coding for the major protein of drosophila larval serum. Genetics 93: 663-679
24. Akam ME, Roberts DB, Richards GP and Ashburner M (1978) Drosophila: the genetics of two major larval proteins. Cell 13: 215-225

25. Akam ME, Roberts DB and Wolfe J (1979) Drosophila hemolymph proteins: purification, characterization, and genetic mapping of larval serum protein 2 in D. melanogaster. Biochem Genet. 16: 101-119

26. Warren JT, Petryk A, Marques G, Jarcho M, Parvy JP, Dauphin-Villemant C, O'Connor MB and Gilbert LI (2002) Molecular and biochemical characterization of two P450 enzymes in the ecdysteroidogenic pathway of Drosophila melanogaster. Proc. Natl. Acad. Sci. USA 99: 11043-11048

27. Petryk A, Warren JT, Marques G, Jarcho MP, Gilbert LI, Kahler J, Parvy JP, Li Y, Dauphin-Villemant C and O'Connor MB (2003) Shade is the Drosophila P450 enzyme that mediates the hydroxylation of ecdysone to the steroid insect molting hormone 20-hydroxyecdysone. Proc. Natl. Acad. Sci. USA 25: 13773-13778

28. Freeman MR, Dobritsa A, Gaines P, Segraves WA and Carlson JR (1999) The dare gene: steroid hormone production olfactory behavior and neural degeneration in Drosophila. Development 20: 4591-4602

29. Buszczak M, Freeman MR, Carlson JR, Bender M, Cooley L and Segraves WA (1999) Ecdysone response genes govern egg chamber development during mid-oogenesis in Drosophila. Development 126: 4581-4589

30. Carney GE and Bender M (2000) The Drosophila ecdysone receptor $(E c R)$ gene is required maternally for normal oogenesis. Genetics 154: 1203-1211

31. Touhara K and Prestwich GD (1993) Juvenile hormone epoxide hydrolase. Photoaffinity labeling purification and characterization from tobacco hornworm eggs. J. Biol. Chem. 268: 19604-19609

32. Harvie PD, Filippova M and Bryant PJ (1998) Genes expressed in the ring gland, the major endocrine organ of Drosophila melanogaster. Genetics 149: 217-231

33. Myster DL, Bonnette PC and Duronio RJ (2000) A role for the DP subunit of the E2F transcription factor in axis determination during Drosophila oogenesis. Development 127: 3249-3261

34. Suzanne M, Irie K, Glise B, Agnes F, Mori E, Matsumoto K and Noselli S (1999) The Drosophila p38 MAPK pathway is required during oogenesis for egg asymmetric development. Genes Dev. 13: 1464-1474

35. Kumar S, McDonnell PC, Gum RJ, Hand AT, Lee JC and Young PR (1997) Novel homologues of CSBP/p38 MAP kinase: activation substrate specificity and sensitivity to inhibition by pyridinyl imidazoles Biochem. Biophys. Res. Commun. 235: 533-538

36. Meier P, Finch A and Evan G (2000) Apoptosis in development. Nature 407: $796-801$

37. Dorstyn L, Colussi PA, Quinn LM, Richardson H and Kumar S (1999) DRONC, an ecdysone-inducible Drosophila caspase. Proc. Natl. Acad. Sci. USA 96: $4307-4312$

38. Vernooy SY, Copeland J, Ghaboosi N, Griffin E, Yoo SJ and Hay BA (2000) Cell death regulation in Drosophila: conservation of mechanism and unique insights. J. Cell Biol. 150: 69-75

39. Brodsky MH, Nordstrom W, Tsang G, Kwan E, Rubin GM and Abrams JM (2000) Drosophila p53 binds a damage response element at the reaper locus. Cell 101: 103-113

40. Peters M, DeLuca C, Hirao A, Stambolic V, Potter J, Zhou L, Liepa J, Snow B, Arya S, Wong J, Bouchard D, Binari R, Manoukian AS and Mak TW (2002) Chk2 regulates irradiation-induced, p53-mediated apoptosis in Drosophila. Proc. Natl. Acad. Sci. USA 99: 11305-11310

41. Hay BA, Wassarman DA and Rubin GM (1995) Drosophila homologs of baculovirus inhibitor of apoptosis proteins function to block cell death. Cell 83 : 1253-1262

42. Tewari M, Yu M, Ross B, Dean C, Giordano A and Rubin R (1997) AAC-11 a novel cDNA that inhibits apoptosis after growth factor withdrawal. Cancer Res. 57: 4063-4069

43. Yin CH, Zou BX, Li MF and Stoffolano Jr JG (1994) Discovery of a midgut peptide hormone which activates the endocrine cascade leading to oogenesis in Phormia regina (Meigen). J. Insect Physiol. 40: 283-292

44. Tatar M, Koplman A, Epstein D, Tu MP, Yin CM and Garofalo RS (2001) A mutant Drosophila insulin receptor homologue that extends life-span and impairs neuroendocrine function. Science 292: 107-110

45. Staveley BE, Ruel L, Jin J, Stambolic V, Mastrinardi FG, Heitzler P, Woodgett JR and Manoukian AS (1998) Genetic analysis of protein kinase B (AKT) in Drosophila. Curr. Biol. 7: 599-602 
46. Clyde D and Bownes M (2000) The Dstpk61 locus of Drosophila produces multiple transcripts and protein isoforms, suggesting it is involved in multiple signaling pathways. J. Endocrinol. 167: 391-401

47. Bownes M, Dübendorfer A and Smith T (1984) Ecdysteroids in adult male and female of Drosophila melanogaster. J. Insect Physiol. 30: 823-830

48. Bownes M (1986) Expression of the genes coding for vitellogenin (Yolk protein). Ann. Rev. Entomol. 31: 507-531

49. Laundrie B, Peterson JS, Baum JS, Chang JC, Fileppo D, Thompson S and McCall K (2003) Germline cell death is inhibited by P element insertions in the $d c p-1 /$ pita nested gene pair in Drosophila. Genetics 165: $1881-1888$

50. Peterson JS, Barkett M and McCall K (2003) Stage-specific regulation of caspase activity in Drosophila oogenesis. Dev. Biol. 260: 113-123

51. Tu MP, Yin CM and Tatar M (2002) Impaired ovarian ecdysone synthesis of Drosphila melanogaster insulinlike receptor mutans. Aging Cell 1: 158-160
52. Soller M, Bownes M and Kübli E (1997) Mating and sex peptide stimulate the accumulation of yolk in oocytes of Drosophila melanogaster. Eur. J. Biochem. 243: 732-738

53. Quackenbush J (2002) Microarray data normalization and transformation. Nat. Genet. 32: 496-501

54. Tautz D and Pfeifle C (1989) A non-radioactive in situ hybridization method for the localization of specific RNAs in Drosophila embryos reveals translation control of the segmentation gene huncback. Chromosome 98: 81-85

55. King C (1970) The meotic behavior of the Drosophila oocyte. Int. Rev. Cytol. 28: $125-168$

56. Hodgetts RB, Clark WC, O'keefe SL, Schouls M, Guild GM and Kala L (1995) Hormone interaction of Dopa decarboxylase in the epidermis of Drosophila is mediated by the Broad-complex. Development 121: 3913-3922

57. Tzolovsky G, Deng MW, Schitt T and Bownes M (1999) The function of the Broad-complex during Drosophila oogenesis. Genetics 153: 1371-1383 